\title{
Conducting polymer nanocomposites with extremely low percolation threshold*
}

\author{
B M MANDAL \\ Polyner Science Lnit, Indian Associalion for the Cultivation of Science, Jadavpur, Calcutta 700032, Indial \\ MS receivod 13 Fobruary 1998
}

\begin{abstract}
I'lis article gives a brief review of olir work on conducting polyaniline nanocomposites which exhibit extremely low pereolation threshold, ca. $\sim 0.03$ vol. \%. The nanocomposites are essentially blends of nanoparticles of $\mathrm{HCl}$ doped polyaniline (PANL) with conventional polymers. The nanoparficles $(<20 \mathrm{~nm})$ were prepared by sonicating a suspension of sterically stabilized colloidal PAN $[$ particles in solutions of conventional polymers. The sonication process breaks down the stable colloid particles into nanoparticles. The lalter are unstable and they aggregate fractally to yield nanoconuosites with extremely low percolation threshold.
\end{abstract}

Keywords. Polyanilinc; conducting polymers; blends; nanocomposites; nanoparticles; perbolation threshold.

\section{Introduction}

Conducting polymers $(\mathrm{CP})$ are important emerging materials with the potential of varied applications c.g. in static charge dissipation, electromagnetic shielding, LED displays, gas senkors, biosensors, pressure sensors, corrosion protection of metsls and semiconductors, transistors, light weight barteries, etc (Ellis 1985; Garnier 1989). However, many of the conducting polymers are difficult to process because of their insolubility and infusibility. Blending with conmodity plastics provides a means of overcoming processing difficulties since the blend can be processed as the matrix polymer. Furthermore, blending reduces the cost of the materials. The cost of the neat materials may be prohibitively high. In this respect lower the $\mathrm{CP}$ loading. cheaper will be the blend. Also, blends with low CP loading can be transparent. This property is technologically attractive because it opens up the possibility of using the transparent conducting blends as electrode materials in liquid crystal display devices. They may also find application as bole injecting materials in flexible polyneric LEDs (Heeger et al 1992) and as antistalic coating on microelectronic components. However: blends constituted of randomly dispersed conducting polymer particles fillers in a nonconductive matrix can reach percolation threshold $\left(f_{\mathrm{p}}\right)$ at a composition of ahout 16 vol.\% of $\mathrm{CP}$ according to the percolation theory (Zallen 1983). Below this eritical concentration the blend is an insulator but the conductivity increases sharply at $f_{\mathrm{f}}$ die to the first development of connectivity between the particles in three-dimension

*MRSI Medal Lecture, Mimbai, 1997 which provides the conducting pathway for the electrical charge carriers. With further increase in concentration of $\mathrm{CP}$ more such conducting paths are formed and the conductivity increases. However, the above nientioned concentration is high enough to make the blend nontransparent and cost-ineffective.

Recently, we have used nanoparticles of CPs as particulate fillers in conventional polyners and achieved percolation at extremely low $\mathrm{CP}$ concentration ca. 0.03 vol\% (Banerjee and Mandal 1995a,b). The fractal aggregation of the unstable nanoparticles has made possible achieving this remarkable result. Our rcsult for polyaniline nanoparticle blends will be presented here.

\section{Experimental}

Dispersions of polyaniline in water were prepared using the method of oxidative dispersion polymerization of atiline at $\sim 0^{\circ} \mathrm{C}$ in acidic aqueous $(1.2 \mathrm{M} \mathrm{HCl})$ medium using armmonium persulfate (APS) initiator oxidant and poly(vinyl methyl ether) (PVME) as polymeric stabilizer (Banerjee et al 1994, 1995). The oblong shaped particles $(250 \times 190 \mathrm{~nm})$ were isolated by centrifugation and purified by way of redispersion in aqueous $\mathrm{HCl}$ Pollowed by centrifugation. For the preparation of the blends the PAVI particles as prepared by the above mentioned method were suspended in solutions of con. ventional polymers in THF, acetone, water etc depending on the solubility of the polymers. The suspension was then placed in a sonicating bath for periods ungine from 1 to $2 \mathrm{~h}$. The resultant dispersions were then cast into films which were tormed on evaporation of the 
solvent. The blend films thus obtained were dried in vacuum at room temperature (Banerjee and Mandal 1995a, b).

The conductivity of the films was measured by the usual four-probe method. For transmission electron microscopy (TEM), the blend film was cast over carbon coated copper grids from suitably diluted dispersions and exarnined using a JEOL JEM 100CT electron microscope.

\section{Nanoparticles of polyaniline}

The polyaniline colloid particles prepared as mentioned above have one remarkable property in that they undergo disintegration when suspension of the particles is sonjcated (figure 1). Figure Ia shows the original PANI particles $(250 \times 190 \mathrm{~nm})$ and figure $1 \mathrm{~b}$ shows that the

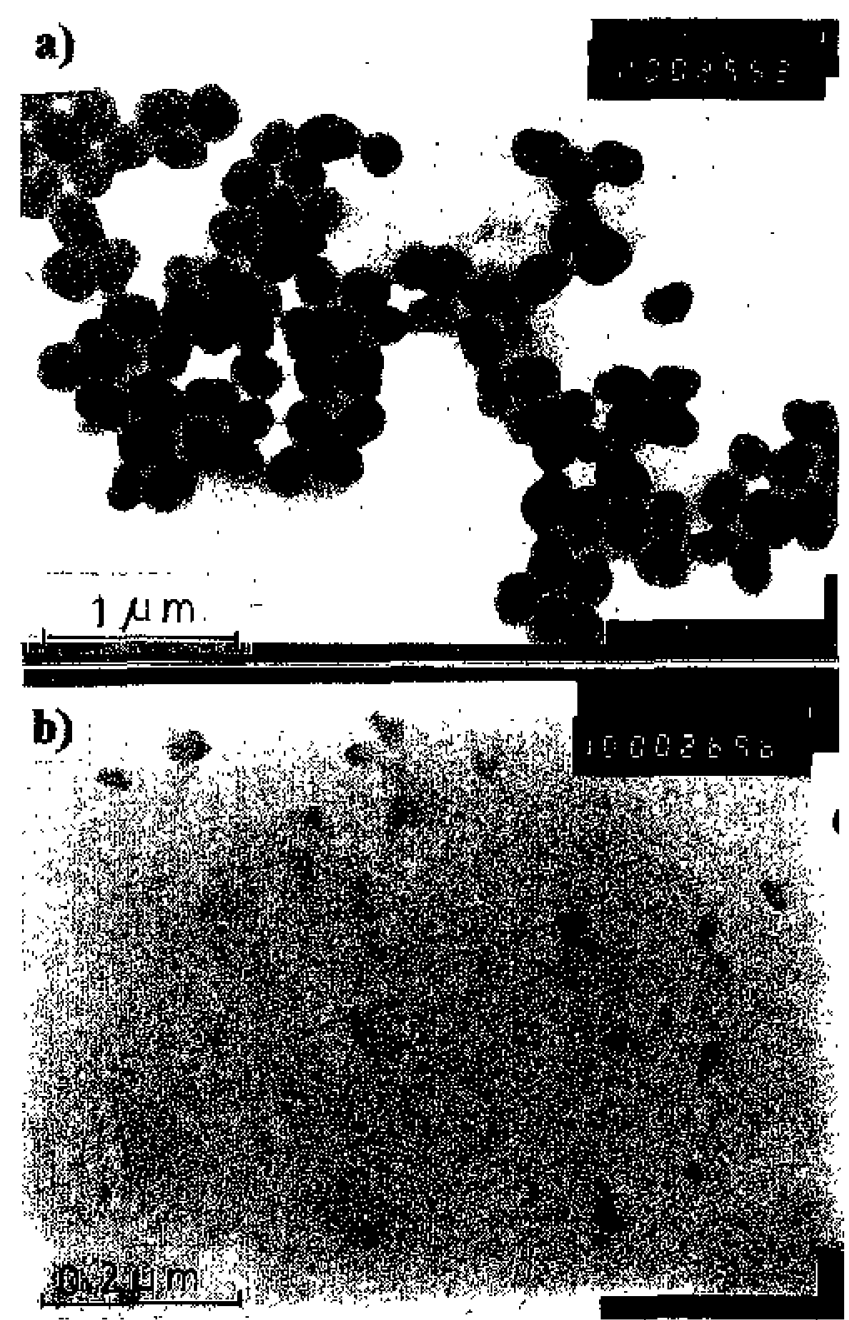

Figure 1. TEM images of PANI $\mathrm{HCl}$ colloid particles: (a) prior to sonication and (b) $] .5$ h after sonication (reprinted with permission ftom Bancriee and Mandal 1995a; 01995 Antcricar Chemical Society). particles have been reduced to sizes less than $20 \mathrm{~nm}$ following sonication.

\section{Blends of polyaniline nanoparticles with conventional polymers}

\subsection{Electrical conductivity}

The electrical conductivity of the blends of the nanoparticles of PANI with conventional polymers when plotted against PANI concentration does not exhibit any well defined percolation threshold as is shown in figure 2 for polyaniline-poly (vinyl alcohol) (PANI-PVA) blends. However, an estimate of the percolation threshold may be obtained by fitting the data used in figure 2 to the scaling law of percolation theory which is as follows (Zallen 1983)

$$
\sigma=C\left(f-f_{\mathrm{p}}\right)^{\prime}
$$

where $C$ is a constant, t the critical exponent, $f$ the volume fraction of the filler particles (here PANI), the subscript p signifying percolation threshold. The fitting is shown in figure 3 which yields a value of $3.6 \times 10^{-4}$ for $f_{p}$ and 1.96 for $t$. For blends using other polyner naterials similar $f_{p}$ and $t$ values were obtained as shown

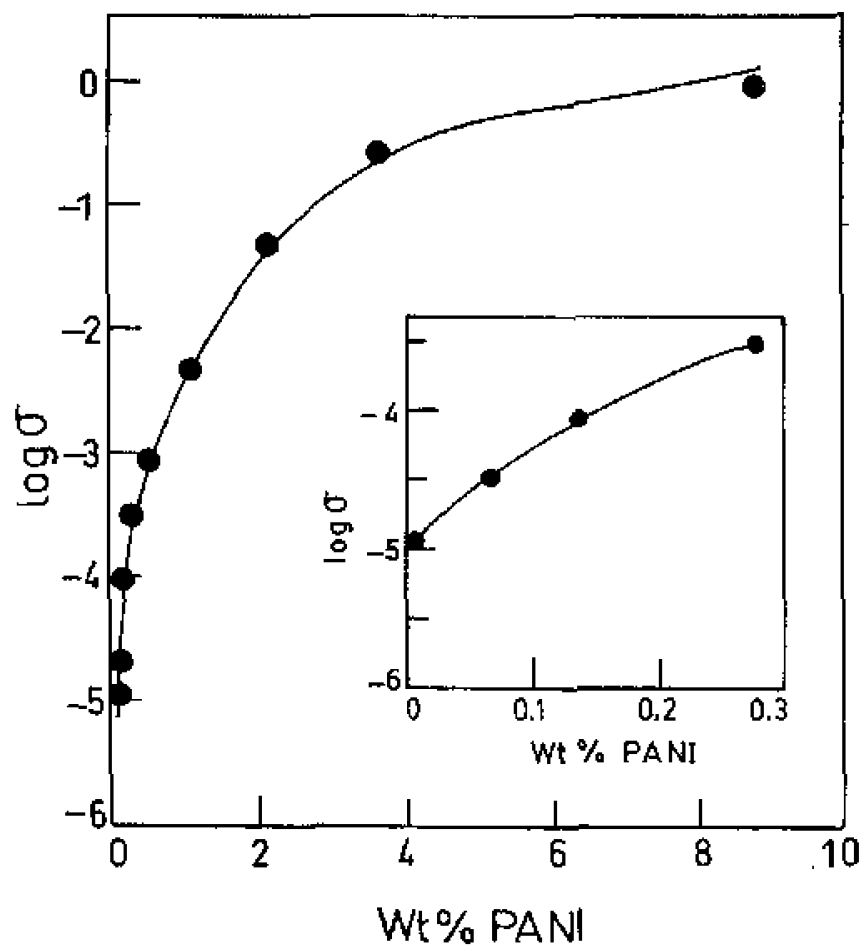

Figure 2. Plot of log clectrical conductivity vs PANI concentration in PANJ-PVA blend lilms. The inset shows an enlarged view for the 10w PANI concentration region (reprinted with permission from Banefee and Mandal 1995a: 1995 American Chemical Sociery). 
in table 1. The $f_{p}$ values are extremely low in comparison to the values of 0.16 predicted by the percolation theory for percolation in three dimension of spherical conducting particles in an insulating matrix.

Previously, Reghu et al (1994) reported conducting polyaniline blends which also have very low $f_{t}$ (ca. $\left.f_{p}=3 \times 10^{-3}\right)$. In contrast to the present method those blends were prepared by the solution blending method. However, in order to make PANI soluble the dopant anion was changed from $\mathrm{Cl}^{-}$to camphor sulphonate (CSA) (Yang et al 1993). The dispersed PANI. CSA phase in the blends were shown with the help of TEM to have a fractal structure. The latter was presumed to result from the self-assembly of PANI - CSA molecules during liquid-liquid phase separation which occurs on the evaporation of solvent from the solutions of the blends (Reghu et al 1993).

Table 1. $f_{p}$ and $t$ data for various blends of PANI $+\mathrm{HCl}$ with conventional polymers.*

\begin{tabular}{lll}
\hline Matrix & $10^{4} f_{\mathrm{p}}$ & $t$ \\
\hline PVC & 4.02 & 1.87 \\
PS & 4.19 & 1.91 \\
PVAC & 3.18 & 1.94 \\
PMMA & 2.14 & 1.89 \\
PVA & 3.60 & 1.96 \\
\hline
\end{tabular}

*Reptinted with permission from Banerjee and Mandal 1995 (c) 1995 American Chemical Society.

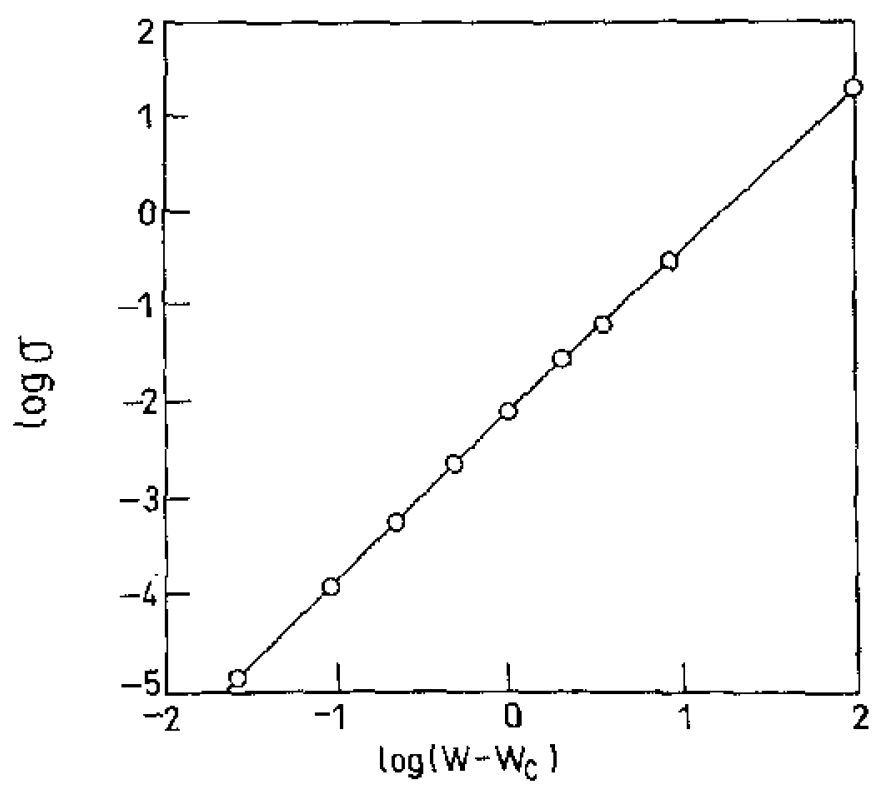

Figure 3. Plot of $\log$ conductwity ys $\log (w-w)$ where $w$ is the wt\% of PANI in the blends and $w_{c}$ that at percolation threshold (reprinled with permission from Banerjee and Mandal 1995a; 6 1995 American Chemical Society).

\subsection{Morphology of the dispersed PANI phase}

In order to find out the origin of the low $f_{p}$ in the present blends the morphology of the dispersed PANI phase was studied with the help of TEM. Figures $4 a-d$ show the bright field images of the PANI-PVA blend films containing respectively $0.035,0.045,0.05$, and 0.5 wt\% of PANI. The images show a fibrillar morphology of the dispersed phase. At the lowest PANI loading of $0.035 \%$ the PANI nanoparticles are formed to exist in isolated clusters. On increasing the PANI, concentration to $0.045 \%$ the clusters appear to be weakly interconnected (figure $4 b$ ) which indicates that the system is near percolation. The structure is stringy and tenuous at this stage which is the characteristics of percolation threshold. As the PANI concentration is increased further to $0.05 \%$ the network becomes more dense. These results thus show that the percolation threshold is somewhere around $0.045 \%$ which is in very good agreement with the $f_{\mathrm{p}}$ determined from conductivity studies (figures 2 and 3 ).

On increasing PANI concentration still further to $0.5 \%$ the system is found to undergo self ordering generating a particular paltern for the network which is in the process of formation in figure $4 \mathrm{~d}$ and near completion in figure 4e. This self-ordering is characteristic of fractal growth Mandelbrot 1983; Peitgen and Richter 1986). The fractal dimension of the structure shown in figure 4e has been determined to be 1.78 (Mandal 1998). The low percolation threshold is attributed to the fractal growth of the dispersed phase. A fractal object grows faster than an Euclidian object since not every point which is defined by the Euclidian space is occupied in a. Fractal object.

\subsection{Mode of aggregation of nanoparticles}

As regards the cause behind the aggregation of the nanoparticles it may be noted that the nanoparticles as produced by the disintegration of the bigger particles do not have enough stabilizer to cover their surface. They are thetefore unstable and it is well known that unstable colloid particles undergo fractal aggregation (Forrest and Witten 1979; Weitz and Oliveria 1984; Lin et al 1990). For example, Weitz and Oliveria (1984) found that stabilized gold sols containing particles of $-14.5 \mathrm{~nm}$ dia. undergo fractal aggregation to form aggregates which have a fractal dimension of -1.75 . In the present case for the structure shown in figure $4 \mathrm{e}$, we determined a fractal dimension of 1.78 (Mandal 1997).

\section{Conclusion}

This study shows that polyaniline nanoparticles can be produced through the disintegration of sterically stabilized 

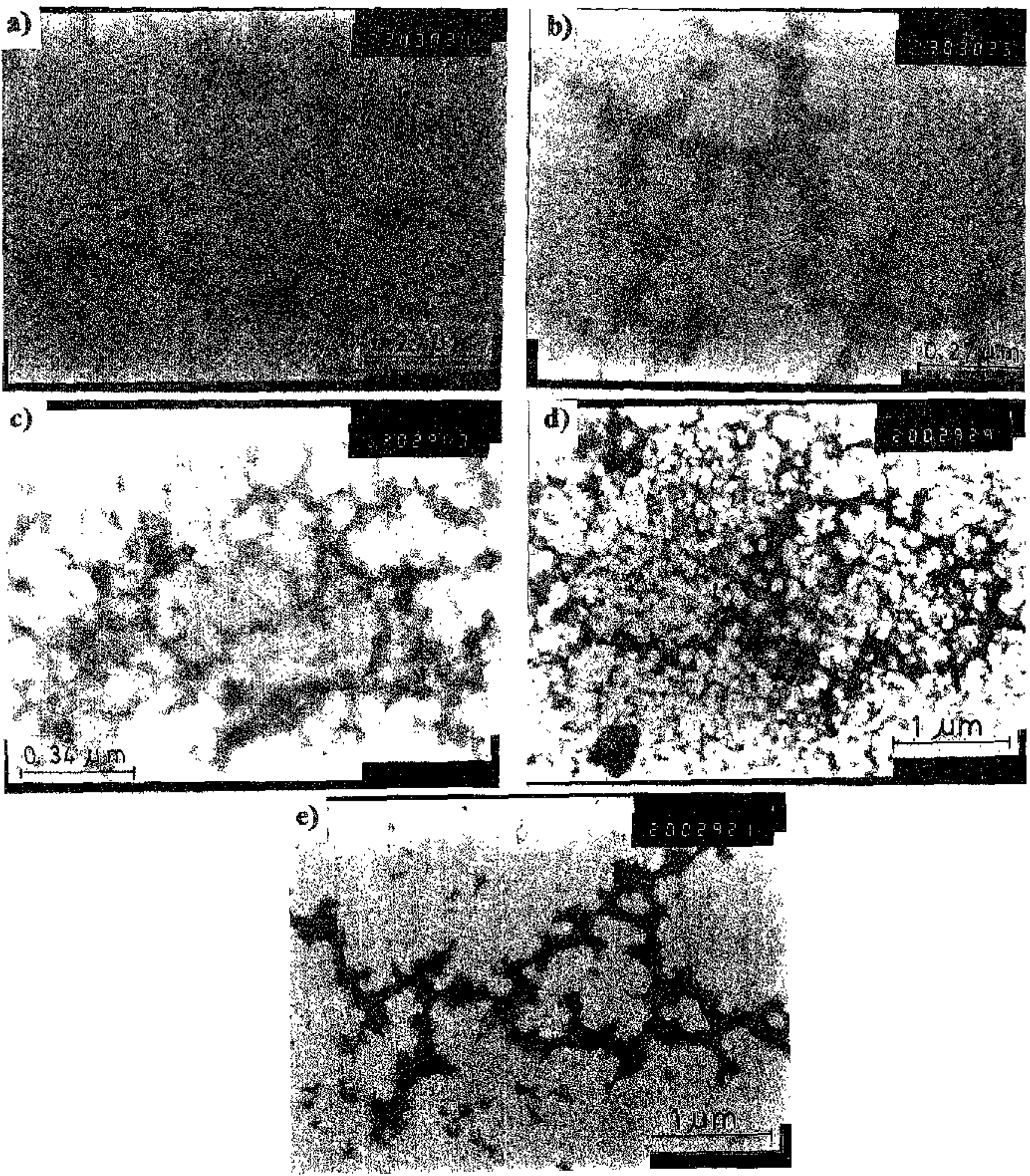

Figure 4. TEM irrages of PANI-PVA blend Films containing different amounts of PANI (in wt\%) (a) 0.135\%, (b) 0.045\%, (c) $0.05 \%$, (d) $0.5 \%$ and (e) $0.5 \%$. but from at different region of the same film as was used for part (d) (reprintcd with pernission from Banerjec and Mandal 1995a; 1995 Ancrican Chemical Society). 
polyaniline colloid particles with the help of ultrasound. Nanoparticles produced in situ in solutions of conventional polymers undergo fractal aggregation such that the blends exhibit extremely low percolation threshold $\left(\sim 3 \times 10^{-4}\right.$ volume fraction).

\section{References}

Banerjee P and Mandal B M 1995a Macromolecules 283940

Banerjee $\mathrm{P}$ and Mandal B M 1995b Synth Met. 74257

Banerjee $\mathrm{P}$, Digar $\mathrm{M} \mathrm{L}$, Bhaltacharyya $\mathrm{S} \mathrm{N}$ and Mandal $\mathrm{B}$ M 1994 Eur. Polym. J. 30499

Banerjee P, Bhallacharyya S N and Mandal B M 1995 Longmuir 112414

Ellis J E 1980 Hondbook on conducting polymers (ed.) $\mathrm{T} \mathrm{J}$ Skotheim (New York: Marcel Dekkerj yol, 1, pp. 489, 501

Forrest S R and Witten Ir T A 1979 J. Phys. A12 L109
Gamier F 1989 Angew. Chem. Int. Ed Eng. 28513

Heeger A J 1992 J. Appl. Phys. Leth 602711

Lin M $Y_{2}$ Lindsay $H$ M, Woitz D A, Klein R, Ball R C and Meakin $\mathrm{P}$ 1990 J. Phys. Cond. Mater 23093

Mandelbro B B 1983 The fractal geometry of nature (New York: Freeman)

Mandal B M 1998 J. Indian Chem. Soc. 75

Peitgen $\mathrm{H} \mathrm{O}$ and Richtel $\mathrm{P} H 1986$ The beatry of froctals (Springer)

Reghu M, Yoon C O, Yang C Y, Moses D, Heeger A $J$ and Cao Y 1993 Macromolecules 267245

Reghu M, Yoon C O, Yang C Y, Moses D. Smith P. Cao Y and Heeger A J 1994 Phys. Rev. B50 13931

Weitz. D A and Otiveria M 1984 Phys. Rev. Lett, 521433

Yang C Y, Cao Y, Smith P and Heeger A J 1993 Syntit. Met. 53293

Zallen R 1983 The physics of amorphots solids (New York: Wiley) ch. 4 